\title{
MECHANISM FOR X-RAY PRODUCTION IN EXTARS
}

\author{
O. P. MANLEY \\ American Science and Engineering* \\ and \\ S. OLBERT \\ Massachusetts Institute of Technology
}

This presentation attempts to describe in very qualitative terms a theory of production of high energy radiation (soft and hard X-rays) in magnetoactive plasmas of astrophysical interest. Special emphasis has been placed on the application of our model to extars and in particular to Sco X-1. More rigorous arguments may be found elsewhere [1] and the interested reader is urged to consult that reference for more details.

Because the construction of our model for X-ray production is independent of evolutionary considerations, in the body of the paper we have steered clear of such admittedly important problems as the origin and nature of the forces holding an extar together. Nor have we dealt adequately with the ultimate source of energy in extars. Our guiding philosophy has been somewhat akin to that taken by an earlier generation of astrophysicists in the context of stellar structures. It will be recalled that there too was an 'insurmountable problem', that of the energetics of stellar luminosities. Nevertheless the major results for a large class of stellar structures were obtained by applying rather elementary physical concepts. To this day, they stand relatively unchanged by the discovery of nuclear synthesis as the source of stellar radiation. It is quite possible that the principles governing the origin and dynamics of extars as well as other new and strange objects in galactic and extragalactic space, such as quasars and pulsars, lie outside of contemporary physics. If that is so, then these recent astronomical discoveries are bound to serve as a stimulus for a new synthesis in physics, much as other, long past astronomical discoveries led to past syntheses. Nonetheless it is tempting to apply some simple ideas stemming from contemporary physics, and see how well one can account for the observed properties of extars to date. It is this course that we propose to explore below.

For reasons dealt with at great length elsewhere [1] our point of departure is that the bulk of extar radiation, viz. X-rays, is due to synchrotron emission by relativistic electrons in a preexisting magnetic field. For the present purposes we assume that the energy required to account for the luminosity of extars is that lodged in the magnetic field. In this context, it is irrelevant whether or not the energy content of this field is replenished by a more fundamental, underlying celestial body - here this consideration would merely change the estimate of the effective lifetime of typical X-ray sources. What is important, however, is that a magnetic field is the only feasible means of storing significant amounts of energy in space without encountering unduly large

* Present address: Visidyne, Inc., 169 Merrimac Street, Woburn, Mass. 01801, U.S.A. 
opacity over a large ( 10 decades) photon energy range. And almost all observations of the known galactic X-ray sources militate against unduly large optical thicknesses.

In the study of ultrarelativistic (UR) electrons with nearly isotropic distribution, as in the case of cosmic rays [2], we are concerned with the equation for the differential number density, $N(E, \mathbf{x}, t)$ of the UR electrons. Here $N d^{3} x \mathrm{~d} E$ represents the number of UR electrons with energies between $E$ and $E+\mathrm{d} E$ within the volume element $d^{3} x$ at position $\mathbf{x}$ and at time $t$. To the extent that we may disregard convection and diffusion in ordinary space we disregard the $\mathbf{x}$-space dependence of $N$. One can then show that $N$ obeys the following Fokker-Planck Equation $[1,2]$ :

$$
\frac{\partial N}{\partial t}=\frac{\partial^{2}}{\partial E^{2}}(D N)+\frac{\partial}{\partial E}\left[\left(W-\left\langle\frac{\Delta E}{\Delta t}\right\rangle\right) N\right],
$$

where $W$ represents the average rate of the energy loss due to dissipative forces, here assumed to be restricted to synchrotron radiation losses; and the average rate of energy gain $\langle\Delta E / \Delta t\rangle$ is related to the 'diffusion coefficient' $D$ by

$$
\left\langle\frac{\Delta E}{\Delta t}\right\rangle=D \frac{d}{\mathrm{~d} E}\left[\ln \left(E^{2} D\right)\right] .
$$

On substituting Equation (2) in Equation (1) and rearranging terms we find the corresponding diffusion equation in energy space:

$$
\frac{\partial N}{\partial t}=\frac{\partial}{\partial E}\left\{E^{2}\left[D \frac{\partial}{\partial E}\left(\frac{N}{E^{2}}\right)+W\left(\frac{N}{E^{2}}\right)\right]\right\} \text {. }
$$

If $N$ is a sufficiently slowly varying function of time we may set Equation (3) equal to zero. Thence, a double integration with respect to the energy yields the quasi-steady state differential number density

$$
N=\text { const } E^{2} \exp \left(-\int \frac{W}{D} \mathrm{~d} E\right) .
$$

The first integration constant is easily shown to vanish for the condition of conservation of particles which we take to be valid here.

If $N$, as given above, is to be valid for a wide variety of non-thermal radiation sources, including extars, the ratio $W / D$ must have certain easily determined attributes insofar as its energy dependence is concerned.

Thus in order for $N$ to be effectively cut-off beyond some critical energy, $W / D$ could be in part a polynomial in energy. On the other hand, to achieve some flexibility in the low energy behavior of $N$ (i.e. energy dependence other than quadratic), and especially to obtain negative spectral power indices characteristic of non-thermal sources, a necessary and sufficient condition on $W / D$ is that it contains as an additive term a member inversely proportional to energy. Inasmuch as the mean energy loss is limited here to synchrotron radiation, $W \sim E^{2}$. Hence a possible form for the diffusion coefficient $D$ is

$$
D \sim \frac{E^{3}}{P_{3}(E)}
$$


where $P_{3}(E)$ is a cubic. The polynomial $P_{n}(E)$ could well be of higher order; however more detailed analysis shows that the higher order terms serve merely to cut $N$ off even faster at high energies - their neglect does not seem to affect the generality of our presentation.

We shall now show how the simple considerations presented above lead to a fairly well defined picture of the environment required for generating non-thermal radiation such as that emanating from extars.

To this end it is instructive to recall here Fermi's ideas of the stochastic acceleration of cosmic-ray particles. By putting

$$
\left\langle\begin{array}{c}
\Delta E \\
\Delta t
\end{array}\right\rangle=E \begin{gathered}
u^{2} \\
c^{2} v_{f}
\end{gathered}
$$

we see that $E\left(u^{2} / c^{2}\right)$ represents the net energy gain per 'collision' with a magnetic irregularity moving with speed $u$, and $v_{f}$ is the average rate at which these random 'collisions' take place. (To be more specific, $v_{f}$ represents the rate of effective momentum transfer) [2]. It is readily appreciated that the 'collision' rate, $v_{f}$, is intrinsically related to the statistics of the moving magnetic irregularities - i.e. to the fluctuations in the medium. In practice, a convenient way of describing fluctuations is the so-called power spectrum which gives a measure of the energy content of the fluctuations at a given frequency $\omega$.

Note here that in order for a function of frequency to be a power spectrum of a realizable stochastic process [3], the function must be (a) symmetric in frequency; (b) integrable on the entire real frequency axis; and (c) non-negative for all real frequencies.

Fermi and others have taken $v_{f}$ to be independent of the particle energy, $E$. An appropriate analysis [1] shows that the corresponding fluctuations must be such as to have a power spectrum, $S(\omega) \sim \omega^{-2}$. This is easily understood in the light of Fermi's acceleration mechanism: the particles were assumed by him to encounter the magnetic clouds at relatively infrequent intervals, the actual interaction time being short compared with the time between collisions. This, of course, is very much like the picture usually employed in the kinetic theory of dilute gases, where the gas particles are in effect assumed to undergo impulsive acceleration at random intervals, the intervals being Poisson-distributed. As is well known, the fluctuations characterized by Poisson statistics have in fact associated with them power spectra which at large frequencies behave as $\omega^{-2}$.

A priori there is no reason why the intervals between interactions should always be very much longer than the interaction times themselves. One can easily imagine a situation in which the spacing between magnetic irregularities is comparable with the radius of gyration, $R_{\mathfrak{g}}$, of the particles within the irregularities - here then the interaction time is comparable with the time between interactions, and of the order of $R_{\mathrm{g}} / c$. We can use the inverse of this quantity as a measure of the 'collision' rate $v_{f}$, which then turns out to be inversely proportional to $E$ because $R_{\mathrm{g}} \sim E$. Again an appropriate analysis [1] shows that if $v_{f}$ has such a behavior with energy the corre- 
sponding fluctuations as seen by the accelerated particles must have a power spectrum $S(\omega) \sim \omega^{-1}$.

Remark here that over the range of particle energies for which $v_{f} \sim E^{-1}$, the mean energy gain per unit time $\langle\Delta E / \Delta t\rangle$ is independent of energy. This will obviously tend to produce a much flatter, and considerably harder particle spectrum than that resulting from the conventional Fermi acceleration mechanism in which the energy gain per unit time is proportional to the particle energy. Obviously a fluctuation spectrum which leads to such a novel energy gain $\langle\Delta E / \Delta t\rangle$, if physically realizable, may be of great significance in the study of non-thermal sources of hard radiation such as the extar Sco X-1. It is tempting therefore to pursue these ideas further.

Because of the integrability condition on a realizable power spectrum, $S(\omega)$ cannot maintain $\omega^{-1}$ behavior for all frequencies. This immediately implies the existence of two critical frequencies $\omega_{1}$ and $\omega_{2}$ (take $\omega_{1}<\omega_{2}$ ): below $\omega_{1}, S(\omega)$ must decrease slower than $\omega^{-1}$, and above $\omega_{2}, S(\omega)$ must decrease faster than $\omega^{-1}$. While no further restrictions on $S(\omega)$ for $\omega<\omega_{1}$ appear to be of any consequence, it is found from more detailed analysis [1], that the required low-energy behavior of the UR electron energy distribution, $N$ (see above), will be obtained if $\lim S(\omega)_{\omega \rightarrow \infty} \sim \omega^{-3}$. These arguments in no way preclude the possibility of a spectral region $\omega$, somewhere between where $S(\omega) \sim \omega^{-1}$ and where $S(\omega) \sim \omega^{-3}$, on which $S(\omega) \sim \omega^{-2}$, or some other intermediate behavior. However, for our present purposes and especially for the sake of analytic simplicity, we ignore such details (see Figure 1).

One may now inquire into the nature of the medium, and in particular, into the

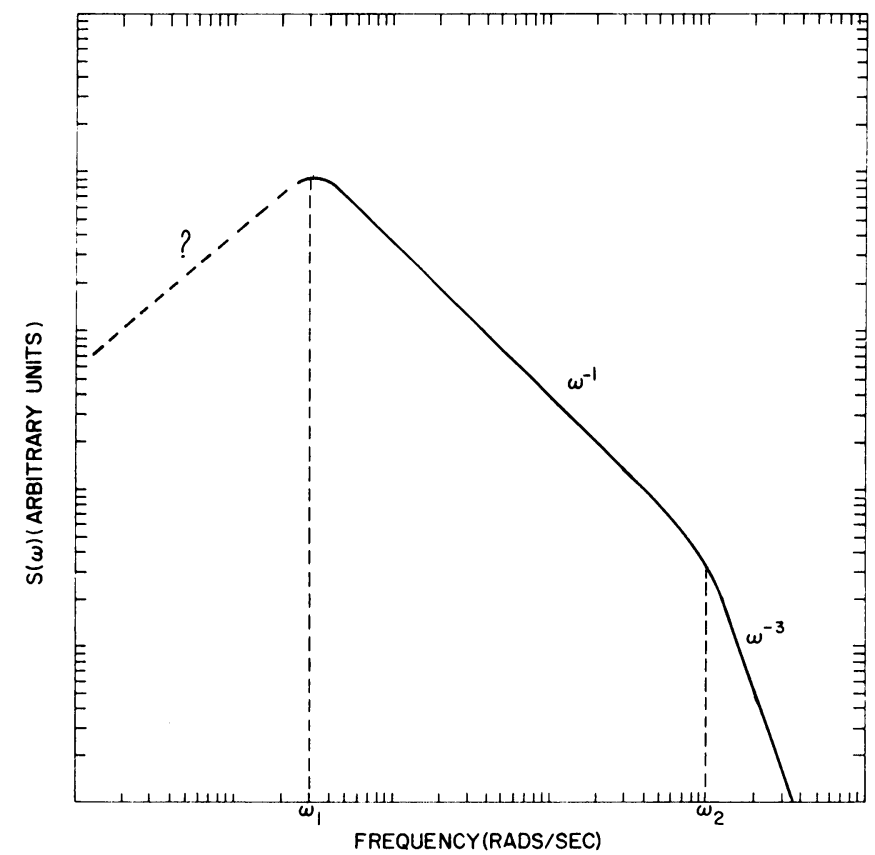

Fig. 1. Power spectrum of magnetic fluctuations. 
fluctuations therein, whose power spectrum might be just the one deduced above from our simple arguments. Of special significance appears to be the presence of the two critical frequencies $\omega_{1}$ and $\omega_{2}$ which endow the power spectrum $S(\omega)$ with the earmarks of band-limited noise. Now if we insist on the stochastic acceleration to arise in virtue of particle interactions with randomly moving magnetic irregularities, we should take into account that such irregularities are resolvable into a superposition of random MHD waves. Thus $S(\omega)$ may be interpreted as precisely the power spectrum of those waves (for simplicity we limit our discussion here to purely transverse Alfvén waves). There occurs then the difficulty that in an unbounded uniform domain MHD waves propagate without attenuation, thus in effect precluding the possibility of generating band-limited MHD noise.

A plausible way out of this dilemma offers itself if we abandon the idea of magnetoactive plasmas being uniform on an arbitrarily large scale. In particular, theoretically useful results are obtained if plasma inhomogeneities are such as to form an aggregate of very long and thin plasmoids. For then it is evident that the finite length and thickness of these plasmoids impose long and short wavelength restrictions on transverse MHD waves. To visualize this, picture the excitation of transverse waves on a finite piece of string - on the one hand, the impossibility of sustaining identifiable vibrations with wavelengths much larger than the length $L_{\|}$of the string itself is easily appreciated; on the other hand, it is equally easy to appreciate the difficulties in sustaining purely transverse deformations with characteristic le ngthsshorter than the diameter of the string, $L_{\perp}$. Since the propagation velocity of the MHD waves in the plasmoids, the Alfvén velocity $u$, is wavelength independent, we find that the finite dimensions of the plasmoids impose low and high frequency cut-offs, $\omega_{1} \simeq u / L_{\perp}$ and $\omega_{2} \simeq u / L_{\|}$, respectively.

Why should the plasma inhomogeneities be characterized by the two lengths $L_{\|}$ and $L_{\perp}$ ? Are these lengths uniquely relatable to any known or empirically determinable plasma properties? The answers to these questions seem to lie in the well-known fact that finite sized, optically thin plasmas which are hot enough to cool by radiation (e.g. free-free or free-bound losses) are unstable with respect to small density (and/or temperature) fluctuations $[4,5]$. This radiation instability is quenched by the large, but finite, thermal conductivity. Dimensional analysis of the interplay between radiation losses, $Q_{\text {rad }}$ - a volume effect, and conduction heat flow, $\kappa \nabla T-$ a surface effect, indicates the existence of a critical length, $L_{c}$, below which the growth of the radiation instability is effectively quenched. Thus

$$
L_{\mathrm{c}}^{2} \approx \kappa T / Q_{\mathrm{rad}}
$$

where $\kappa$ is the thermal conductivity and $T$ is the plasma temperature. In the presence of a strong magnetic field, the flow of heat transverse to the magnetic field is strongly inhibited, while being essentially unaffected along the magnetic field $\left(\kappa_{\|} \approx \kappa\right)$. Thus we expect two critical lengths - one governing the transverse size of a stable plasmoid $-L_{\perp}$, the other its longitudinal size $-L_{\|} \simeq L_{\mathrm{c}}$. They are related by

$$
\left(\begin{array}{c}
L_{\perp} \\
L_{\|}
\end{array}\right)^{2} \simeq \frac{\kappa_{\perp}}{\kappa_{\|}} \simeq \begin{gathered}
v_{\text {coll }} \\
\omega_{b}
\end{gathered}
$$


where $v_{\text {coll }}$ is the effective collision frequency in a plasma, and $\omega_{b} \equiv e B / m_{e} c$ the symbols having their conventional meaning. Here magnetic field is taken to be strong if $v_{\text {coll }} / \omega_{b} \ll 1$. In spite of this limitation the plasma may not be considered as collisionless because our chosen time scale is sufficiently long for effects of radiation to be of explicit importance. We see this in (a) having taken into account the role the radiation losses play in plasma stability and (b) noting that, for instance in the case of free-free radiation, the mean number of elastic collisions between the occurrence of radiative collisions is of the order of $m_{e} c^{2} / \alpha k T$, where $\alpha$ is the fine structure constant.

So far we have paid scant attention to the physical reasons for the details of the behavior of $S(\omega)$ between the two critical frequencies $\omega_{1}$ and $\omega_{2}$, nor have we inquired why the behavior outside of those frequencies should be as stipulated on purely phenomenological grounds. In this respect, for the present we must rest our case on purely empirical grounds - viz. the observation of magnetic noise spectra under at least two widely different conditions : interplanetary magnetic field fluctuations [6] and magnetic noise spectra generated under certain laboratory conditions [7]. In both cases the general features of the measured power spectra are found to be just those postulated by us here. Thus we feel justified in suggesting the universal nature and character of band-limited MHD noise in magneto-active plasmas and in asserting its prevalence under a wide range of astrophysical conditions. Chances are that the detailed behavior of $S(\omega)$ with frequency depends on the statistical ensemble of the plasma properties - e.g. temperature, density and magnetic field - in the region under consideration. This should be so because as we have seen, the bandwidth limitations of vibrations within individual filaments depend on their length and diameter, which in turn depend on the local temperature, density and mean magnetic field. Moreover our considerations have yielded only upper bounds on $L_{\perp}$ and $L_{\|}$- as yet we have no knowledge of the rules and statistics whereby these dimensions are distributed below those bounds.

We are now in a position to write down the explicit quasi-steady state solution to the equation governing the energy dependence of the differential number density $N$. It can be shown that (expressing from here on all relativistic energies in terms of the Lorentz factor $\gamma$ ) it takes the form [1]

where

$$
N(\gamma)=\text { const. } \gamma^{2-\zeta} \exp \left\{-\left(\frac{\gamma}{\gamma_{0}}\right)^{2}\left[1+\frac{2}{3 \zeta}\left(\frac{\gamma_{m}}{\gamma_{0}}\right)^{2} \frac{\gamma}{\gamma_{m}}+\frac{\gamma_{m}}{\gamma}\right]\right\},
$$

$$
\begin{aligned}
\zeta & \equiv \frac{10 \omega_{b}}{3 A \omega_{2}^{2}} \frac{r}{c} \\
\gamma_{m} & \equiv \frac{40}{3 \pi} u \omega_{1} \omega_{b} / c \omega_{2}^{2} \\
\gamma_{0}^{2} & \equiv 3 A \frac{u^{2}}{r c \omega_{b}}
\end{aligned}
$$

with $r$ the classical electron radius, $m\left(m_{\mathrm{p}}\right)$ the electron (proton) mass, $n$ the mean 
plasmoid particle density, $c$ velocity of light, $u \equiv\left(B^{2} / 4 \pi n m_{\mathrm{p}}\right)^{1 / 2}$, and

$$
A=\left\langle(\delta B / B)^{2}\right\rangle\left[1-\left(\omega_{1} / \omega_{2}\right)^{2}\right] / 2 \ln \left(\omega_{2} / \omega_{1}\right) .
$$

Note that $\left\langle(\delta B / B)^{2}\right\rangle=\int_{-\infty}^{\infty} S(\omega) \mathrm{d} \omega$ is the relative mean square amplitude of the magnetic field fluctuations and can be thought of as the ratio of the magnetic energy in fluctuations to the mean magnetic field energy. In arriving at $N$ as given above we have modelled $S(\omega)$ in such a way as to simplify analysis, but without sacrificing in any way its principal attributes deduced from the preceding arguments. However, some of the numerical constants may be somewhat sensitive to the particular model picked. This should not affect our major conclusions.

The observed physical attributes of an extar are connected by the three parameters, $\zeta, \gamma_{m}$, and $\gamma_{0}$ defined above. They may seem to arise from a rather arbitrary and not necessarily unique arrangement of various coefficients in arriving at the form of $N(\gamma)$ given above. However, a closer study reveals that they are endowed with potential physical significance hard to overlook.

Thus, on taking into account the relationships between $\omega_{1}, \omega_{2}$ and the properties of the underlying magneto-active plasma, it is found that

$$
\zeta=\frac{400}{A} \frac{m_{\mathrm{p}}}{m \alpha}\left(\frac{3 k T}{2 \pi m c^{2}}\right)^{3 / 2} .
$$

Note that $\zeta$ governs the behavior of the low energy end of the UR electron energy distribution. It seems remarkable that this ultimate result does not depend on the plasma density and magnetic field intensity; rather it is primarily a function of the plasma temperature and the ratio of the mean energy in magnetic field fluctuations to the energy in the mean magnetic field. Of course, underlying our considerations is the assumption that the electron temperature is high enough so that the plasma is nearly fully ionized.

Next we turn to the energy, $\gamma_{m}$. We find that we can write it in a physically more transparent form:

$$
\gamma_{m}=\frac{160}{3 \pi^{2}} \frac{\omega_{1} \omega_{b}}{\omega_{2}} \frac{L_{\perp}}{c}
$$

from which $\gamma_{m}$ is seen to be an energy at which the radius of gyration of an electron is guaranteed to be much smaller than $L_{\perp}$, i.e. smaller than the radius of the filamentary plasmoids which concern us here (this follows from the condition $\omega_{1} \ll \omega_{2}$ implied throughout our considerations). It is perhaps even more remarkable that $\gamma_{m}$ may be shown to be essentially a natural constant corresponding to an energy of $\sim 0.5 \mathrm{GeV}$. One can conjecture that it represents a critical energy for relativistic electrons, such that when it is exceeded, the relaxation of the underlying magnetic field is governed by synchrotron emission losses of the UR electrons, rather than by plasma free-free (free-bound) emission losses.

Finally, we come to $\gamma_{0}$. In the limit of small $\gamma_{m} / \gamma_{0}-$ a limit found to be of some 
consequence below $-\gamma_{0}$ denotes a characteristic energy of the relativistic electrons described by the function $N(\gamma)$. We shall see that $\gamma_{0}$ plays an important role in determining the hardness of the synchrotron spectrum generated by an ensemble of electrons distributed according to $N(\gamma)$.

The function $N(\gamma)$ behaves as a power law for low electron energies, its index lying between -1 and 0 , a range of importance in the study of extars provided

$$
2<\zeta<3
$$

On the other hand, $N(\gamma)$ decreases quite rapidly for $\gamma>\gamma_{0}$. It has been pointed out $[8,9]$ that if $N(\gamma)$ were to be sharply cut-off at some upper energy, the resulting synchrotron spectrum would evince an exponential behavior hardly distinguishable from that emitted by hot, optically thin plasma at an appropriate temperature. As we shall see on comparison with data, the high energy tail of our $N(\gamma)$ approaches adequately such a sharp cut-off.

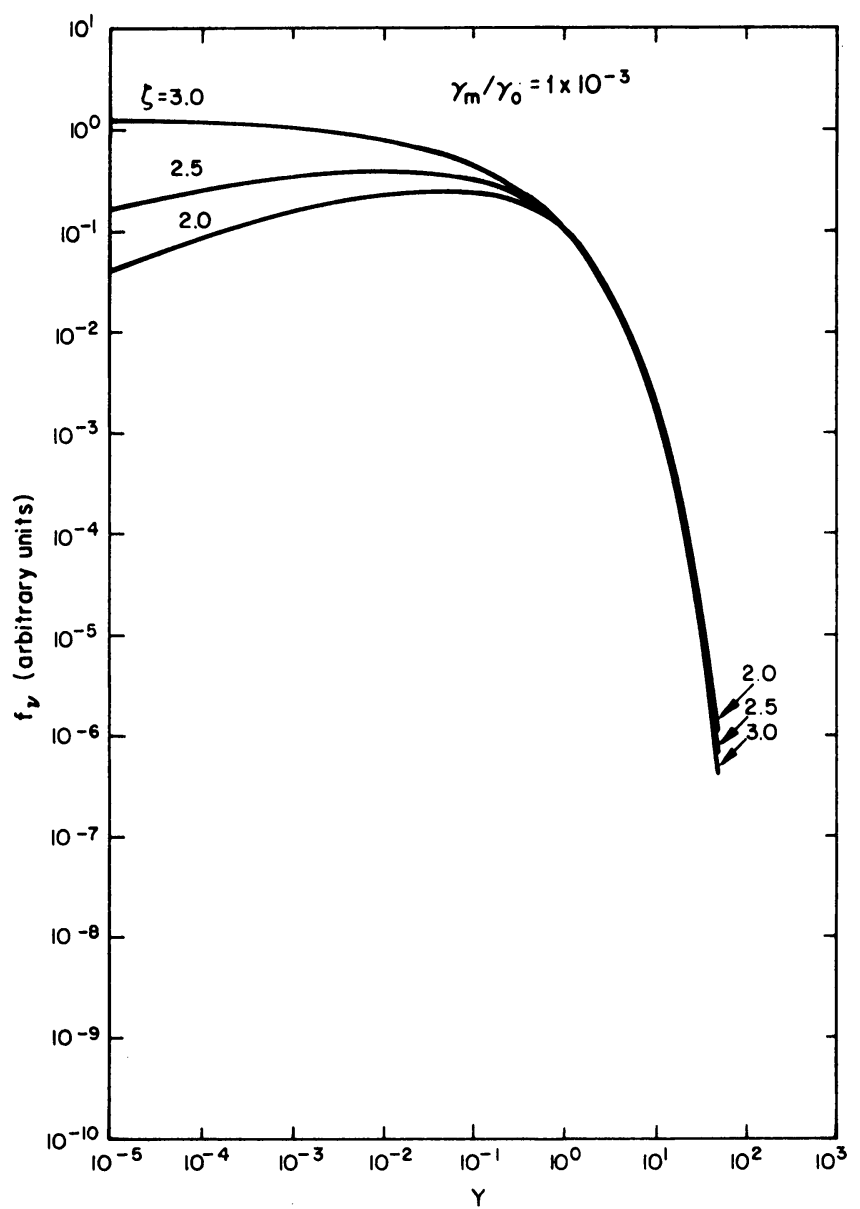

Fig. 2. Dependence of the synchrotron spectrum, $f_{v}$, on $\zeta$ for a fixed $\gamma_{m} / \gamma_{0}$. 
We now form the synchrotron spectrum, $f_{v}$, emitted by UR electrons - distribution with energy according to $N(\gamma)$ :

$$
f_{v}=\operatorname{Ber} \sqrt{3} \int_{0}^{\infty} \mathrm{d} \gamma N(\gamma) \frac{2 x}{3 \gamma^{2}} \int_{2 x / 3}^{\infty} \mathrm{d} \eta K_{5 / 3}(\eta),
$$

where $x \equiv 2 \pi v / \omega_{b}, v$ being the photon frequency.

In practice it turns out that the bulk of the more reliable X-ray astronomy data appears to fall in a region such that

$$
0.1 \lesssim 2 x / 3 \gamma_{0}^{2} \lesssim 10
$$

It is found that in this frequency regime for $\gamma_{m} / \gamma_{0} \lesssim 10^{-1}, f_{v}$ is independent of $\gamma_{m} / \gamma_{0}{ }^{\circ}$ $f_{v}$ for several values of $\zeta$ is shown in Figure 2 .

We now turn to the determination of the specific parameters describing an extar, interpreting available data in the light of the above considerations. We restrict ourselves here to data concerning Sco X-1 principally because this source is the one most extensively studied. For the sake of concreteness in the following numerical estimates we take the distance to Sco X-1 to be $300 p c$ [10] and hence, the X-ray luminosity (i.e. nearly the total luminosity), $L_{x}=5 \times 10^{36} \mathrm{ergs} / \mathrm{sec}$. Further, in the same spirit we assume Sco X-1 to be approximated by a sphere of radius $R$.

Recall first that the low electron energy end of the spectrum depends on the index $\zeta$ which in turn depends on the plasma temperature and mean square amplitude of magnetic fluctuations. Substitution of $T \approx 10^{5} \mathrm{~K}$, and $A \approx 10^{-1}$, (i.e. $\left\langle(\delta B / B)^{2}\right\rangle \approx 1$ ) into Equation (10) yields

$$
\zeta=2.5 \pm 0.5
$$

spanning the indeterminancy in the color of the optical spectrum of Sco X-1 due to both the interstellar reddening and the observed intrinsic color fluctuations. Thus, we see that as a direct consequence of our model the observed 'flat' photon spectrum, implying a hard electron spectrum, is consistent with the observed high excitation lines requiring a plasma temperature on the order of $10^{5} \mathrm{~K}$. It should be noted that the parameters $T$, and $A$, selected here are probably reasonable mean values for Sco X-1 as a whole. Notwithstanding that they are subject to some temporal and spatial variations, which may account directly for the reported amplitude and color changes of the visible continuum as well as for the variability of the equivalent line widths. Of course, the possible radio emission attributed to Sco X-1 is consistent with the range of values of $\zeta$ given above.

On setting

$$
B \gamma_{0}^{2} \approx 6 \times 10^{11} \mathrm{G}
$$

there results a reasonably good fit to the Sco X-1 X-ray spectral data, Figure 3 $[11,12,13]$. Remarkably enough, the fact that $N(\gamma)$ is actually not sharply cut-off above $\gamma_{0}$, serves to harden the predicted photon spectrum just enough to fit the recent 
high energy measurement without additional ad hoc assumptions about the structure of the extar.

Note that the recent report of a measurement of soft X-ray emission from Sco X-1 [12], is consistent with our model if the mean interstellar hydrogen density in the direction of that extar is $\sim 0.5 \mathrm{~cm}^{-3}$ ( $\sim 2.5$ mean free paths for X-ray absorption).

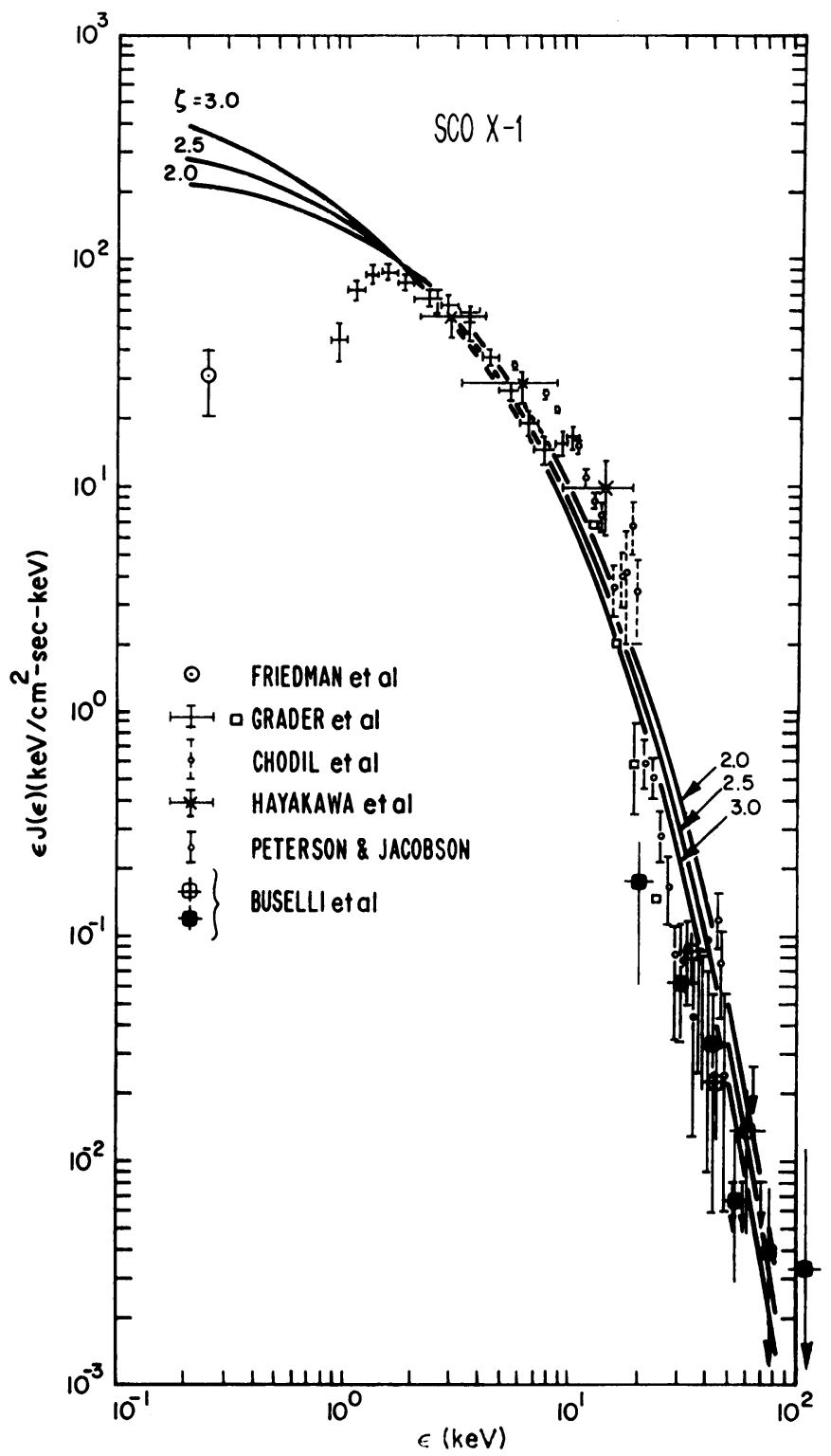

Fig. 3. Comparison of our computations with Gould's (1967) compilation of spectral measurements of Sco X-1. Note (a) that the soft X-ray measurement reported earlier by Friedman has been amended to conform with the latest NRL measurements (Fritz et al., 1968) and (b) new spectral measurements beyond $20 \mathrm{keV}$ by the University of Adelaide group (Buselli et al., 1968) have been added. 
We find further that with the radius $R \sim 100 A U, n \approx 10^{6} \mathrm{~cm}^{-3}$ (consistent with absence of forbidden lines in the optical spectrum of Sco $X-1$ ) and $B \approx 2 G^{*}$, the relativistic electron energy density is

$$
N_{0} E_{\mathrm{UR}} \approx 5 \times 10^{-8} \mathrm{ergs} / \mathrm{cm}^{3}
$$

where $N_{0}$ is the mean density of UR electrons and $\left(E_{\mathrm{UR}}=\gamma_{0} m c^{2}\right)$. Note that for our model

$$
N_{0} E_{\mathrm{UR}} \ll n k T
$$

so that the dynamics of the extar is not affected directly by the relativistic electron pressure.

The spectrum of the magnetic fluctuations in our simple model spans characteristic frequencies which coincide with the observed optical and X-ray relaxation times ranging from seconds to hours. We may then assert plausibly that the observed fluctuations characterizing both the optical and X-ray ends of the Sco X-1 spectrum are a direct consequence of fluctuations in the underlying magnetic field and hence in the underlying plasma density and UR electron density. In accordance with this argument, the absence of significant polarization of the visible light emanating from Sco X-1 may well be attributed to the lack of preferred orientation of the fluctuating magnetic field. The color changes attendant on amplitude fluctuations may be evidence of sporadic hardening of the electron spectrum.

\section{Acknowledgements}

We wish to acknowledge the interest of Drs. M. Annis and R. Giacconi of American Science and Engineering, Inc. in this presentation.

This presentation was supported in part by Air Force Office of Scientific Research (OAR) Contract No. F44620-67-C-0065.

\section{References}

[1] Manley, O. P. and Olbert, S.: 1969, Astrophys. J. to appear.

[2] Ginzburg, V. L. and Svrovatskii, S. I.: 1964, Origin of Cosmic Rays, Pergamon Press, New York.

[3] Lee, Y. W.: 1960, Statistical Theory of Communication, J. Wiley, New York.

[4] Manley, O. P. and Olbert, S.: 1968, presented at Asilomar Conference on Plasma Instabilities in Astrophysics, California, October.

[5] Field, G. B.: 1965, Astrophys. J. 142, 531.

[6] Coleman, P. J.: 1968, Astrophys. J. 153, 371.

[7] Patrick, R. M. and Pugh, E. R.: 1969, Phys. Fluids 12, 366.

[8] Greenstein, J.: 1964, Astrophys. J. 140, 666.

[9] Manley, O. P.: 1966, Astrophys. J. 144, 1253.

[10] Wallerstein, G.: 1967, Astrophys. Letters 1, 31.

[11] Gould, R. J.: 1967, Am. J. Phys. 35, 376.

[12] Fritz, G., Meekins, J. F., Henry, R. C., Byram, E. T., and Friedman, H.: 1968, Astrophys. J. Letters 153, L199.

[13] Buselli, G., Clancy, M. C., Davison, P. J. N., Edwards, P. J., McCracken, K. G., and Thomas, R. M.: 1968, Nature 219, 5159.

* Under the assumption of no external energy input to the magnetic field this corresponds to a lifetime of $\sim 40 \mathrm{yrs}$. 